\title{
Violencia comunitaria en adolescentes desfavorecidos: exposición, impacto percibido y consecuencias psicológicas
}

\section{Community violence in socioeconomically disadvantaged adolescents: Exposure, perceived impact, and psychological consequences}

\author{
Cindy Mels y Lucía Fernández \\ Universidad Católica del Uruguay, Montevideo, Uruguay
}

\begin{abstract}
Resumen: La violencia comunitaria forma parte de la vida cotidiana de cada vez más niños y jóvenes, particularmente en las grandes ciudades. La exposición a este tipo de violencia ha sido asociada con el desarrollo de varios problemas conductuales y emocionales, tales como conductas agresivas y delincuentes, ansiedad y depresión. Este estudio pretende explorar la exposición de adolescentes uruguayos en situación de vulnerabilidad socioeconómica a la violencia comunitaria y su impacto percibido, e investigar su relación con problemas internalizantes y externalizantes reportados por ellos. Se consiguió una muestra de 101 adolescentes de 13 a 18 años en la ciudad de Montevideo, pertenecientes a tres centros socioeducativos seleccionados deliberadamente y por conglomerado. Se administró el Hopkins Symptom Checklist para adolescentes, la Escala de Violencia Comunitaria y un cuestionario sociodemográfico. Se encontró que el $84,2 \%$ de la muestra había sido expuesto a la violencia comunitaria. El impacto percibido de la violencia apareció como predictor de problemas internalizantes, junto a la variable edad. Los resultados sugieren que diferentes mecanismos explican los distintos tipos de problemas psicológicos investigados. Se discuten las implicaciones para la intervención y la prevención.
\end{abstract}

Palabras clave: adolescencia, trauma, salud mental, pobreza, violencia comunitaria.
Abstract: Community violence increasingly forms part of the daily lives of children and youth, particularly in large cities. Exposure to this type of violence has been associated with the development of various behavioral and emotional problems, such as aggressive and delinquent behaviors, anxiety and depression. This study aims to explore the exposure of socioeconomically vulnerable Uruguayan adolescents to community violence and its perceived impact, and investigate its relationship with internalizing and externalizing behavior problems. We obtained a sample of 101 adolescents aged 13 to 18 years, residing in the city of Montevideo, attending to 3 socio-educational centers purposefully selected and clustered. The Hopkins Symptom Checklist for adolescents, a scale for community violence and a sociodemographic questionnaire were administered. We found that $84.2 \%$ of our sample had been exposed to community violence. The perceived impact of exposure appeared as a predictor of psychological problems, together with the variable age. The results suggest that several mechanisms explain the different kinds of psychological problems investigated. Implications for intervention and prevention were discussed.

Keywords: adolescence, trauma, mental health, poverty, community violence.

Contacto: C. Mels. Av. 8 de Octubre 2733, C.P. 11600, Montevideo, Uruguay. Correo electrónico: cimels@ucu.edu.uy

Cómo citar: Mels, C. y Fernández, L. (2015). Violencia comunitaria en adolescentes desfavorecidos: exposición, impacto percibido y consecuencias psicológicas. Revista de Psicología, 24(1), 1-21.

http://dx.doi.org/10.5354/0719-0581.2015.36863 


\section{Introducción}

El Estudio Global de Homicidios 2013 (UNODC, 2014) identifica a Latinoamérica como la región más violenta a nivel mundial. Por tanto, la violencia forma parte de la vida cotidiana de niños y jóvenes, particularmente en las grandes ciudades, donde se perciben crecientes niveles de violencia y crímenes variados en el espacio público (Steinbrenner, 2010). Este fenómeno es denominado "violencia comunitaria" y comprende la exposición a armas de fuego, cuchillos, drogas y delitos aleatorios, tales como robos, rapiñas, ataques físicos con y sin armas, y homicidios (Kennedy y Ceballo, 2014). Fue reconocido por la OMS (2003) como un problema para la salud pública a escala mundial, particularmente en comunidades económica y socialmente desfavorecidas.

Gracias a los procesos de transición e independización típicos de la adolescencia, los adolescentes suelen realizar más actividades fuera de su hogar y pasar mayor cantidad de tiempo en la calle. Debido a esto, se muestran involucrados más frecuentemente en la violencia comunitaria (Bowen y Bowen, 1999; Rosenfeld, Richman, Bowen y Wynns, 2006; Aisenberg y Herrenkohl, 2008). Aunque mucha atención política y académica ha sido designada a los menores infractores como autores de dichos actos violentos, existe todo un campo de investigación que estudia las consecuencias psicológicas de sus víctimas, relacionando la exposición a la violencia comunitaria en adolescentes -ya sea de forma indirecta, al tratarse de testigos, como de manera directa, al tratarse de la victimización- con problemas emocionales y conductuales (Lynch, 2003; Mels, 2012), así como con fracaso escolar (Borofsky, Kellerman, Baucom, Oliver y Margolin,
2013; Rosenfeld et al., 2006). Evidencia empírica ha demostrado que la victimización en la adolescencia aumenta la probabilidad de conductas criminales y que esta relación se explica, parcialmente, por las alteraciones psicológicas generadas por la exposición a la violencia y las reacciones de afrontamiento asociadas a las mismas (Hartinger-Saunders et al., 2011). Estas conclusiones generan nuevas perspectivas en torno a las causas de la delincuencia adolescente y la prevención de su reproducción, y destacan la importancia del estudio de víctimas adolescentes de la violencia comunitaria (Cooley-Strickland et al., 2009).

Consecuencias psicológicas de la exposición a la violencia comunitaria

En particular, se han observado síntomas relacionados con el Trastorno de Estrés Postraumático (TEPT), siendo altas las tasas de este trastorno en adolescentes víctimas (Horowitz, McKay y Marshall, 2005; Lynch, 2003) y testigos de violencia comunitaria (Guerra, Huesmann y Spindler, 2003; Saltzman, Compas, Connor-Smith, Harding Thomsen y Wadsworth, 2001).

También es frecuente la incidencia de problemas internalizantes como la depresión, la ansiedad y las quejas somáticas (Copeland-Linder, Lambert e Ialongo, 2010; Ludwig y Warren, 2009; Salzinger, Feldman, Stockhammer y Hood, 2002). No obstante, dichos síntomas internalizantes han sido menos investigados, dejando muchas interrogantes acerca de su relación con la exposición a la violencia comunitaria (Fowler, Tompsett, Braciszewski, Jacques-Tiura y Baltes, 2009). Algunos autores han conectado este cuadro específico de síntomas con la exposición continua y prolongada a actos de violencia comunitaria 
(Cooley-Strickland et al., 2009; Fowler et al., 2009) y la coincidencia con otras fuentes de estrés, como los problemas económicos (Mels, 2012; Salzinger et al., 2002).

Varios estudios informan sobre la alta prevalencia de conductas agresivas, desafiantes y abuso de sustancias problemas externalizantes, según la taxonomía de Achenbach y Edelbrock (1978)- en adolescentes víctimas y testigos de violencia comunitaria (CopelandLinder et al., 2010; Leiner et al., 2012; Ludwig y Warren, 2009; Osypuk et al., 2012). Teorías de aprendizaje social sugieren que la pertenencia a contextos con altas tasas de violencia, promueve $\mathrm{y}$ normaliza las conductas agresivas a través del modelaje del comportamiento, llevando a que se produzca cada vez más violencia al nivel de la comunidad (Guerra et al., 2003).

La diversidad de síntomas asociados a la exposición a la violencia comunitaria y la fluctuación de su severidad reportada en la literatura, demuestran claramente que las consecuencias de la exposición a este tipo de violencia no son las mismas para todos los adolescentes (Aisenberg y Herrenkohl, 2008). Desde el concepto de la resiliencia -concebido por Luthar y Cicchetti (2000) como un proceso dinámico que tiene como resultado la adaptación positiva en contextos de gran adversidadsurge el interés por el estudio de la interacción entre variables (fisiológicas, cognitivas, familiares, culturales, etc.) que faciliten la adaptación positiva, a pesar de los riesgos, y que ayuden a explicar la variedad de reacciones psicológicas encontradas (Gorman-Smith y Tolan, 1998; Lynch, 2003). Además, de acuerdo con la teoría socioecológica de Bronfenbrenner (1979), las teorías de resiliencia conciben la exposición a la violencia comunitaria como parte del contexto ecológico, donde otros múltiples riesgos, pero también recursos, influyen en el desarrollo humano.

En la última década, se han investigado los factores y mecanismos que moderan la relación entre la exposición a la violencia comunitaria y el desarrollo psicológico. En su revisión de la literatura, Lynch (2003) las cataloga según tres tipos: las características personales del niño, las del ambiente social, y las de la exposición.

En lo que se refiere a los factores personales que moderan los efectos psicológicos de la exposición a la violencia comunitaria, se han estudiado ciertas variables de índole demográfico tales como, sexo, edad, etnia y, aunque mucho menos, de tipo psicológico (temperamento y estilos de afrontamiento). En cuanto a la variable sexo, los varones suelen sufrir niveles más altos de exposición a la violencia comunitaria (CooleyStrickland et al., 2009; Osypuk et al., 2012). No obstante, esta observación no necesariamente implica que su desarrollo psicológico se vea más severamente impactado por la violencia comunitaria, en comparación con las niñas. En cuanto a esto, algunos estudios informan acerca de la mayor prevalencia de problemas psicológicos en mujeres frente a varones, a pesar de los diferentes niveles de exposición (Javdani, Abdul-Adil, Suarez, Nichols y Farmer, 2014). Por otro lado, algunos autores afirman que el impacto de la variable sexo varía en función de la edad y el contexto específico bajo investigación (Cooley-Strickland et al., 2009; Grant et al., 2005; Osypuk et al., 2012).

Siguiendo con la variable edad y retomando lo anteriormente establecido, se ha reconocido su relevancia para explicar los niveles de exposición, siendo lineal y 
positiva la relación entre la edad y la cantidad de eventos violentos experimentados, también dentro de la etapa de la adolescencia (Lambert, Ialongo, Boyd y Cooley, 2005; Fowler et al., 2009). Además, la edad influye en la vulnerabilidad de los adolescentes frente a las consecuencias psicológicas de dicha exposición: mientras que la menor edad está asociada con mayor prevalencia de síntomas internalizantes, los adolescentes mayores suelen presentar más problemas psicológicos en general $\mathrm{y}$ externalizantes en específico, que sus pares más jóvenes (Fowler et al., 2009).

En cuanto al estudio de las características del ambiente social, los factores más estudiados son el nivel socioeconómico y las características de la familia del adolescente. Asimismo, el contexto y la constitución familiar cobran importancia en el impacto de la violencia en el adolescente (Lynch, 2003). La parentalidad adecuada, la cual incluye soporte, monitoreo y comunicación intrafamiliar, actúa como factor protector de sintomatología psicológica reportada en niños y adolescentes expuestos a violencia comunitaria (Hartinger-Saunders et al., 2011). Comparando tipos de familias sobre la base de su constitución, se ha demostrado el papel protector de la familia nuclear en los efectos negativos de la exposición a la violencia (Lynch, 2003; Salzinger et al., 2002). Respecto a lo socioeconómico, se reconoce que la exposición a actos violentos y sus efectos negativos son superiores en contextos desfavorecidos (Gustafsson, Larsson, Nelson y Gustafsson, 2009). Esta elevada vulnerabilidad ha sido explicada por el estrés acumulativo generado por problemas económicos y la exclusión social, aspectos característicos de una vida en pobreza (Mels, Derluyn, Broekaert y Rosseel, 2010a; Miller y Rasmussen, 2010; Seiffge-Krenke, 2000).
En cuanto a las características de la exposición violenta, se destaca la importancia de la acumulación de eventos violentos experimentados, lo cual muestra una relación positiva con la cantidad y severidad de problemas psicológicos reportados (Lambert et al., 2005; Mels, Derluyn, Broekaert y Rosseel, 2009). Por otro lado, se ha investigado la medida en que los dos grandes tipos de exposición - la directa como víctima y la indirecta como testigo- generan efectos diferenciales en la salud mental. Aunque se ha sugerido que la mayor proximidad física a los eventos violentos (la victimización directa) produciría mayores niveles de sintomatología, los resultados del metaanálisis de 114 estudios en torno a la temática (Fowler et al., 2009) demuestran que esto solamente se aplica a la prevalencia de problemas internalizantes y externalizantes, pero no diferencia en cuanto a la prevalencia de síntomas de TEPT. No obstante, Kennedy y Ceballo (2014) advierten que -a pesar de los esfuerzos hechos para desenredar los efectos específicos de diferentes tipos de exposición- en el contexto de la violencia comunitaria urbana, la exposición suele ser acumulativa y variada, y no se deja investigar de forma aislada. En contraste, otros autores han sugerido la relevancia de investigar el impacto percibido de la exposición a la violencia, aparte de sus características objetivas (Hill y Madhere, 1996; Lynch, 2003).

\section{El papel de la percepción del impacto de la violencia comunitaria sobre sus consecuencias psicológicas}

La teoría de evaluación cognitiva (Lazarus y Folkman, 1984) plantea que el impacto y las consecuencias psicológicas que genera la exposición a eventos estresantes, se determina parcialmente por la percepción que tiene el individuo de di- 
chos eventos. Por tanto, a pesar de la consistente relación establecida entre la frecuencia o acumulación de eventos violentos experimentados y sintomatología psicológica, es probable que la percepción que tiene el individuo acerca de los eventos tenga mayor valor explicativo. La eventual comprobación de esta hipótesis generaría implicancias importantes para la prevención de problemas emocionales y conductuales tras la exposición a la violencia, ya que permite generar iniciativas para moldear el significado atribuido a los eventos, con el objetivo de estimular reacciones resilientes, a pesar de la exposición sufrida.

No obstante, hasta donde se sabe, solamente dos estudios han indagado específicamente en el efecto de la percepción de la violencia comunitaria. En primer término, nos referimos al estudio de $\mathrm{Ai}$ senberg y Herrenkohl (2008), con 137 adolescentes norteamericanos. De los actos de violencia comunitaria sufridos por los sujetos, el acto percibido como más grave en $74 \%$ de los casos no correspondía al acto considerado más grave según criterios objetivos (basados en el código penal y el promedio de pena al que los autores son condenados). En cuanto a su relación con síntomas de TEPT, fue el evento objetivamente más grave el que impactó más en ellos, no el evento percibido como el más grave.

Para el caso del segundo estudio, investigando el rol del impacto percibido de la violencia comunitaria sobre problemas internalizantes y externalizantes, Hill y Madhere (1996) llegaron a otra conclusión. En su investigación con 150 niños afroamericanos, encontraron que la percepción de la violencia comunitaria estaba más fuertemente relacionada con síntomas de ansiedad, conductas disruptivas y competencia social reducida, que con la exposición real a la violencia comunitaria.

Queda claro que se necesitan más evidencias para poder profundizar en el entendimiento acerca del papel de la percepción de la violencia comunitaria, frente a la exposición real, en el desarrollo de sus efectos nocivos.

\section{Objetivo del estudio e hipótesis}

El objetivo de este estudio es explorar, en una muestra de adolescentes uruguayos, la exposición a la violencia comunitaria y su impacto percibido por un lado, e investigar su relación con problemas internalizantes y externalizantes reportados por ellos.

En un esfuerzo por aislar esta relación entre exposición a la violencia comunitaria, su impacto percibido y problemas internalizantes y externalizantes, los análisis estadísticos de este estudio mantienen controladas ciertas variables de riesgo conocidas en la literatura (a nivel individual, edad y sexo; a nivel contextual la constitución familiar). El nivel socioeconómico (otra variable contextual) se mantiene controlado por la composición de la muestra, la cual incluye solamente adolescentes de nivel socioeconómico bajo pertenecientes al área metropolitana de Montevideo.

Siguiendo la teoría de evaluación cognitiva esperamos que la relación establecida entre la exposición a la violencia comunitaria y los niveles de problemas internalizantes y externalizantes reportados se vea parcialmente mediada por el efecto de su impacto percibido. En cuanto a las variables edad, sexo y constitución familiar -aunque no formen parte del enfoque principal de este estudio- se espera una mayor vulnerabilidad de mu- 
jeres, adolescentes mayores, y un efecto protector de la familia nuclear, sobre los puntajes de problemas psicológicos.

\section{Metodología}

\section{Escenario del estudio}

Aunque Uruguay debe ser considerado como un país atípico en la región, en el análisis de sus tasas de delitos entre 1985 y 2007, Paternain y Sanseviero (2008) concluyeron que aumentó la frecuencia de delitos contra personas, homicidios, lesiones, denuncias contra la propiedad, delitos sexuales, rapiñas y daños, entre otros, siendo el porcentaje más alto en zonas con desempleo alto y pobreza intensificada. Por otro lado, Munyo (2012) señala que esta evolución afecta desproporcionalmente a la población de adolescentes: están cada vez más involucrados como autores y víctimas de violencia. En una encuesta reciente (Trajtenberg y Eisner, 2015), en una muestra de 2185 alumnos de 14 a 18 años de liceos públicos y privados en la ciudad de Montevideo, se encontró que $24.6 \%$ de la muestra había sido víctima de, al menos, uno de los tres tipos de violencia indicados (robo, agresión y violencia sexual) en los últimos 12 meses. El delito más frecuentemente reportado fue el robo (17.9\%), seguido por la agresión (11\%) y el abuso sexual violento $(1.4 \%)$.

\section{Participantes}

Se llevó a cabo un estudio transversal ex post facto en tres centros socioeducativos en zonas de contexto socioeconómico bajo en la ciudad de Montevideo, los cuales ofrecen apoyo pedagógico gratuito a adolescentes. Los centros fueron seleccionados en función de su ubicación (aspirando a una mayor dispersión a través de las zonas de nivel socioeconómico bajo en la ciudad) y contactos institucionales preestablecidos. Un total de 103 adolescentes de entre 12 y 18 años participaron en el estudio. Dos de los participantes fueron excluidos de la muestra por haberse notado un sesgo en sus respuestas, por lo que la muestra final se compuso de 101 adolescentes, con promedio numérico de edad 15.15 años $(D E=$ 1.88). Las características de la muestra están resumidas en la tabla 1 . Se destaca la escasa cantidad de mujeres en la muestra $(n=16)$ frente a varones $(n=85)$.

Tabla 1

Características sociodemográficas de la muestra

\begin{tabular}{llcc}
\hline Variables & Grupo & Frecuencia $(N)$ & Porcentaje $(\%)$ \\
\hline Sexo & Mujer & 16 & 15.8 \\
& Varón & 85 & 84.2 \\
Edad & $12-15$ años & 54 & 53.5 \\
& $16-18$ años & 47 & 46.5 \\
$\begin{array}{l}\text { Constitución } \\
\text { familiar }\end{array}$ & Familia nuclear & 54 & 53.5 \\
& & & \\
& Familia & 22 & 21.8 \\
& extendida/ensamblada & & 14.9 \\
& Familia monoparental & 15 & 8.9 \\
\hline
\end{tabular}




\section{Instrumentos}

Escala de Violencia Comunitaria. Esta escala fue generada sobre la base de datos cualitativos obtenidos en una encuesta llevada a cabo en el año 2011 con 529 adolescentes montevideanos de variado nivel socioeconómico (Mels y Trías, 2014). Evalúa la exposición (sí-no) a cuatro experiencias de violencia comunitaria relevantes para el contexto en estudio, incluyendo vivencias como testigo (agresión física) y víctima (robo, agresión física, amenaza), a través de los últimos 12 meses. Asimismo, evalúa el impacto percibido de cada uno de los acontecimientos (a través de la pregunta: "¿Cómo te ha impactado emocionalmente?"), los cuales se deben evaluar del 1 al 4 en una escala de poco a mucho. Se calcula el total de la violencia comunitaria objetiva, sumando la cantidad de eventos violentos reportados por cada participante. El total de la violencia percibida consiste en el promedio del impacto percibido de todos los eventos reportados por los participantes.

\section{Hopkins Symptom Checklist para} adolescentes (versión uruguaya, HSCL-A). El Hopkins Symptom Checklist para adolescentes (HSCL-A) (Bean, Derluyn, Eurelings-Bontekoe, Broekaert y Spinhoven, 2007; adaptado y validado por Mels y Trías, 2014) es una escala que mide síntomas basados en los criterios del Manual diagnóstico y estadístico de los trastornos mentales, (DSM-IV, en inglés) para depresión y ansiedad, agrupándose en una subescala de comportamientos internalizantes (24 ítems), y para los trastornos disocial y negativista desafiante, y abuso de sustancias (subescala de problemas externalizantes, 12 ítems). Los ítems -adaptados a la vida adolescenteevalúan la severidad de los síntomas en una escala Likert de 1 a 4 ( 1 = nunca, $2=$ $a$ veces, $3=$ seguido, $4=$ siempre $)$. La es- cala está disponible en 21 idiomas con valores adecuados de validez de constructo, contenido y criterio, obtenido en una muestra de adolescentes inmigrantes y autóctonos holandeses y flamencos (Bean et al., 2007), y adolescentes afectados por la Guerra en el Congo (Mels, Derluyn, Broekaert y Rosseel, 2010b). El instrumento fue adaptado para uso en adolescentes uruguayos, mostrando características psicométricas aceptables (consistencia interna, estructura factorial y validez convergente) en una muestra de 529 adolescentes montevideanos de variado nivel socioeconómico (Mels y Trías, 2014), y comparables con las características anteriormente establecidas (Bean et al., 2007; Mels et al., 2010b). Cuenta con valores de Alpha de Chronbach de .90 para la escala total, .90 para la subescala internalizante y .75 para la subescala externalizante.

Cuestionario sociodemográfico. Se les solicitó a los participantes datos relativos a su edad y sexo. En cuanto a la variable constitución familiar, se pidió a los adolescentes que describieran con quiénes vivían, información que fue codificada por los investigadores en una de cuatro categorías conforme a las utilizadas por el Instituto Nacional de Estadística en Uruguay (INE, 2013):

Familia nuclear: familia tipo con hijos, es decir, la compuesta por padre, madre e hijos.

$\sim$ Familia extendida o ensamblada: aquellas familias en las cuales se ha ausentado alguno de sus miembros y otros se han anexado.

$\sim$ Familia monoparental: familias en las que solo uno de los padres está presente.

Otros: quienes viven solos o a cargo de un tutor, es decir, alguna persona que no es ni su madre ni padre biológico. 


\section{Procedimiento}

Todos los adolescentes que asisten a los centros socioeducativos seleccionados fueron invitados a participar a través de una carta informativa acerca del estudio, dirigida a ellos y a sus padres, acompañada por un formulario de consentimiento informado.

A continuación, se procedió a la administración colectiva de los cuestionarios a los adolescentes en los meses de julio y agosto del año 2013. La administración se realizó en los salones de clase, en el horario curricular, y tuvo una duración de treinta minutos aproximadamente.

El procedimiento para este estudio -enmarcado en una línea de investigación con procedimiento establecido- fue aprobado por el comité de ética de la Facultad de Psicología de la Universidad Católica del Uruguay.

\section{Análisis}

Contando con una distribución normal de los puntajes del HSCL-A en la muestra (el estadístico de prueba Shapiro-Wilk presenta un valor de .067 que corresponde a un nivel de significación de .093), se utilizaron pruebas paramétricas para los análisis inferenciales, aplicando un nivel de confianza de $p \leq .05$ como umbral para la significancia estadística (con excepción de las correlaciones). Para el análisis de los datos se utilizó el programa estadístico SPSS versión 18.0 (2009).

Tras la exploración descriptiva de la violencia comunitaria reportada y su impacto percibido para toda la muestra, se continuó con la comparación de medias de las variables exposición a la violencia comunitaria (EVC, el total de los eventos de violencia comunitaria reportados), total impacto percibido (TIP), y los puntajes de problemas internalizantes y externalizantes. Se utilizó la prueba $t$ de Student para los grupos bicategóricos (sexo, grupo etario), y ANOVA unidireccional para la variable constitución familiar, complementado por la prueba post hoc de Tukey.

Se usó ANCOVA para investigar cómo las variables independientes (categóricas y continuas) están asociadas con los síntomas psicológicos. El modelo ANCOVA incluyó todos los efectos directos de las variables: sexo, grupo etario, constitución familiar, violencia comunitaria acumulada e impacto percibido acumulado, y los efectos de interacción de las variables categóricas con las continuas. Se usó $\eta^{2}$ como estimación del tamaño de efecto.

\section{Resultados}

\section{Violencia comunitaria y problemas internalizantes y externalizantes}

En la tabla 2 se muestra la frecuencia de los actos de violencia comunitaria sufridos y el promedio del impacto correspondiente a los mismos (en una escala de 1 a 4 ).

Se observa que $79.2 \%$ de los adolescentes en la muestra $(n=80)$ informa haber sido testigo de la violencia física contra otra persona, siendo el evento más frecuentemente reportado. A la vez es el evento con mayor promedio de impacto subjetivo reportado $(M=2.32 ; D S=$ 1.08). En comparación, los ítems vinculados a la victimización de violencia comunitaria cuentan con frecuencias más bajas $y$, sorprendentemente, con promedios inferiores de impacto percibido. 
Tabla 2

Frecuencia y promedio del impacto de los tipos de eventos de violencia sufridos

\begin{tabular}{lccc}
\hline $\begin{array}{l}\text { Eventos de violencia } \\
\text { comunitaria reportados }\end{array}$ & $\begin{array}{c}\text { Sí } \\
N(\%)\end{array}$ & $\begin{array}{c}\text { No } \\
N(\%)\end{array}$ & $\begin{array}{c}\text { Impacto percibido } \\
\text { por ítem* } M(D T)\end{array}$ \\
\hline $\begin{array}{l}\text { 1. Te han robado } \\
\text { 2. Te han pegado, atacado o }\end{array}$ & $22(21.8 \%)$ & $78(77.2 \%)$ & $1.57(1.08)$ \\
lastimado & & & $1.83(1.05)$ \\
$\begin{array}{l}\text { 3. Te han amenazado } \\
\begin{array}{l}\text { 4. Has visto lastimar, pegar o } \\
\text { atacar a otra persona }\end{array}\end{array}$ & $80(79.2 \%)$ & $21(20.8 \%)$ & $2.32(1.08)$ \\
\hline
\end{tabular}

*Calculado sobre la base de los participantes que reportaron haber sufrido el evento correspondiente.

Los datos en cuanto a la acumulación de eventos violentos sufridos se presentan en la tabla 3. Solamente el $15.8 \%(n=$ 16) de la muestra informa no haber sufrido ninguno de los eventos de violencia comunitaria evaluados. De los 85 participantes $(84.2 \%)$ que reportaron la exposición a eventos de violencia comunitaria, 46 (equivalente al $45.6 \%$ de la muestra total) se han encontrado expuestos a varios eventos de forma acumulativa.

Tabla 3

\section{Violencia comunitaria acumulada}

\begin{tabular}{cc}
\hline $\begin{array}{c}\text { Cantidad de eventos } \\
\text { reportados }\end{array}$ & $N(\%)$ \\
\hline 0 & $16(15.8)$ \\
1 & $39(38.6)$ \\
2 & $28(27.7)$ \\
3 & $14(13.9)$ \\
4 & $4(4.0)$ \\
\hline
\end{tabular}

La tabla 4 informa acerca del promedio del impacto total percibido por la muestra, y los puntajes obtenidos en las escalas del HSCL-A. Al no contar con normas validadas al respecto, están me- ramente presentados aquí de modo referencial para la muestra total.

Tabla 4

Media y desviación típica de las medidas continuas

\begin{tabular}{lc}
\hline Medidas & $M(D T)$ \\
\hline $\begin{array}{l}\text { Total exposición a } \\
\text { violencia comunitaria } \\
\text { (EVC) }\end{array}$ & $1.474(1.064)$ \\
$\begin{array}{l}\text { Total impacto } \\
\text { percibido (TIP) }\end{array}$ & $1.886(1.088)$ \\
$\begin{array}{l}\text { HSCL-A escala } \\
\text { internalizante }\end{array}$ & $1.727(0.997)$ \\
$\begin{array}{l}\text { HSCL-A escala } \\
\text { externalizante }\end{array}$ & $1.578(0.911)$ \\
HSCL-A escala total & $1.680(0.885)$ \\
\hline
\end{tabular}

Comparación por sexo, edad y constitución familiar

La tabla 5 presenta las medias (con desviación estándar) de las variables EVC, TIP y problemas psicológicos internalizantes y externalizantes según sexo, grupos de edad y constitución familiar, así como las pruebas inferenciales para su comparación. 
Tabla 5

Eventos de violencia, impacto percibido y problemas psicológicos según sexo, edad y constitución familiar

\begin{tabular}{|c|c|c|c|c|c|c|}
\hline Variable & $\begin{array}{l}\text { Grupo - prueba } \\
\text { de comparación }\end{array}$ & EVC & TIP & HSCL-A Total & $\begin{array}{c}\text { HSCL-A } \\
\text { Internalizante }\end{array}$ & $\begin{array}{c}\text { HSCL-A } \\
\text { Externalizante }\end{array}$ \\
\hline \multirow[t]{3}{*}{ Sexo } & Varón & $1.62(D S 1.07)$ & $3.32(D S 2.43)$ & $1.50(D S 0.25)$ & $1.51(D S 0.29)$ & $1.49(D S 0.35)$ \\
\hline & Mujer & $0.94(D S 0.68)$ & $1.80(D S 1.37)$ & $1.58(D S 0.32)$ & $1.71(D S 0.42)$ & $1.33(D S 0.2)$ \\
\hline & $t$ de Student & $\begin{array}{l}t(99)=3.33 \\
p=0.002\end{array}$ & $\begin{array}{l}t(92)=2.34 \\
p=0.02\end{array}$ & $\begin{array}{l}t(99)=-1.08 \\
p=0.281\end{array}$ & $\begin{array}{l}t(99)=-1.83 \\
p=0.083\end{array}$ & $\begin{array}{l}t(99)=2.59 \\
p=0.014\end{array}$ \\
\hline \multirow[t]{3}{*}{ Edad } & 12-15 años & $1.39(D S 1.05)$ & $2.84(D S 2.04)$ & $1.45(D S 0.26)$ & $1.51(D S 0.32)$ & $1.35(D S 0.24)$ \\
\hline & 16-18 años & $1.66(D S 1.03)$ & $3.34(D S 2.66)$ & $1.58(D S 0.24)$ & $1.58(D S 0.31)$ & $1.60(D S 0.38)$ \\
\hline & $t$ de Student & $\begin{array}{l}t(99)=-1.30 \\
p=0.196\end{array}$ & $\begin{array}{l}t(99)=-1.03 \\
p=0.305\end{array}$ & $\begin{array}{l}t(99)=-2.63 \\
p=0.010\end{array}$ & $\begin{array}{l}t(99)=-1.08 \\
p=0.285\end{array}$ & $\begin{array}{l}t(99)=-3.93 \\
p=0.000\end{array}$ \\
\hline \multirow{4}{*}{$\begin{array}{l}\text { Constitución } \\
\text { familiar }\end{array}$} & Familia nuclear & $1.35(D S 0.97)$ & $2.73(D S 2)$ & $1.46(D S 0.28)$ & $1.56,(D S 0.96)$ & $1.36(D S 0.30)$ \\
\hline & $\begin{array}{l}\text { Familia } \\
\text { ensamblada/ } \\
\text { extendida }\end{array}$ & $\begin{array}{l}1.45 \\
(D S 0.96)\end{array}$ & $\begin{array}{l}2.95 \\
(D S 2.17)\end{array}$ & $\begin{array}{l}1.54 \\
(D S 0.24)\end{array}$ & $\begin{array}{l}1.55 \\
(D S 0.27)\end{array}$ & $1.55(D S 0.32)$ \\
\hline & $\begin{array}{l}\text { Familia } \\
\text { monoparental }\end{array}$ & $\begin{array}{l}2.13 \\
(D S 1.12)\end{array}$ & $\begin{array}{l}4.27 \\
(D S 3.03)\end{array}$ & $\begin{array}{l}1.56 \\
(D S 0.22)\end{array}$ & $\begin{array}{l}1.55 \\
(D S 0.27)\end{array}$ & $\begin{array}{l}1.60 \\
(D S 0.34)\end{array}$ \\
\hline & ANOVA & $\begin{array}{l}F(2,88)=3.64 \\
p=0.300\end{array}$ & $\begin{array}{l}F(2,82)=2.69 \\
p=0.074\end{array}$ & $\begin{array}{l}F(2,88)=0.57 \\
p=0.566\end{array}$ & $\begin{array}{l}F(2,88)=0.01 \\
p=0.990\end{array}$ & $\begin{array}{l}F(2,88)=5.24, \\
p=0.007\end{array}$ \\
\hline
\end{tabular}


En lo que refiere a los grupos de edad, a nivel descriptivo se observa una mayor EVC en el grupo de adolescentes de mayor edad, y un mayor TIP en los adolescentes menores, aunque estas diferencias en función de edad no resultan estadísticamente significativas. En cuanto a los problemas psicológicos reportados en estos grupos, los puntajes son significativamente más altos en adolescentes mayores, tanto en la escala total del HSCLA como en la subescala externalizante, siendo estos últimos más destacados aún.

Por último, en cuanto a la variable constitución familiar, no se encuentran diferencias en la EVC según grupo, ni en el total del TIP. No obstante, respecto de los problemas psicológicos evaluados, se destaca la diferencia significativa en cuanto a los puntajes obtenidos en la escala externalizante $(F(2,88)=5.24 ; p$ $=0.007)$. Las pruebas post hoc revelan diferencias significativas en los puntajes obtenidos en la escala externalizante, por un lado, entre adolescentes pertenecientes a familias nucleares y los pertenecientes a familias monoparentales (Media de la diferencia $=-0.241$; Error estándar $=0.090 ; p=0.024) \mathrm{y}$, por otro lado, entre aquellos pertenecientes a familias nucleares y familias extendidas/ensambladas (Media de la diferencia $=0.191 ;$ Error estándar $=0.078$; $p=0.044)$. No obstante, los adolescentes de familias monoparentales y los de familias extendidas no muestran diferencias significativas entre sí (Media de la diferencia $=0.051 ;$ Error estándar $=$ $0.104 ; p=0.877)$.

\section{Relación entre la exposición objetiva y percibida y problemas psicológicos}

La tabla 6 presenta de modo exploratorio las correlaciones bivariadas de Pearson entre las variables centrales del estudio: exposición objetiva y percibida, y problemas psicológicos. Se destaca la fuerte correlación positiva entre EVC y TIP. El TIP muestra una correlación (débil a moderada) con todas las escalas del HSCL-A, mientras que la EVC se ve únicamente correlacionada (de forma moderada) con la escala de conductas externalizantes.

De forma conclusiva y presentado en la tabla 7 , se investigó la asociación lineal de las variables EVC y TIP, sexo, edad y constitución familiar, con las escalas del HSCL-A, a través de un modelo ANCOVA. Este modelo -operado para las tres variables dependientes de forma separada (escala total, internalizante y externalizante)- incluyó los efectos tanto directos como de interacción de las distintas variables.

Tabla 6

Correlaciones

\begin{tabular}{lllll}
\hline & EVC & HSCL-A total & $\begin{array}{l}\text { HSCL-A } \\
\text { externalizante }\end{array}$ & $\begin{array}{l}\text { HSCL-A } \\
\text { internalizante }\end{array}$ \\
\hline TIP & $0.816^{*}$ & $0.444^{*}$ & $0.424^{*}$ & $0.306^{*}$ \\
EVC & - & $0.278^{*}$ & $0.369^{*}$ & 0.142 \\
\hline
\end{tabular}

* La correlación se considera significativa a un nivel de 0.01 . 
Tabla 7

Análisis de covarianza

\begin{tabular}{|c|c|c|c|c|c|c|c|c|c|}
\hline & \multicolumn{3}{|c|}{$\begin{array}{c}\text { Problemas } \\
\text { internalizantes }\end{array}$} & \multicolumn{3}{|c|}{$\begin{array}{c}\text { Problemas } \\
\text { externalizantes }\end{array}$} & \multicolumn{3}{|c|}{ Escala total HSCL } \\
\hline & $F$ & $P$ & $\eta^{2}$ & $F$ & $P$ & $\eta^{2}$ & $F$ & $p$ & $\eta^{2}$ \\
\hline Sexo & 4.082 & .047 & .055 & .000 & .990 & .000 & 3.355 & .071 & .046 \\
\hline $\begin{array}{l}\text { Edad en } \\
\text { categorías }\end{array}$ & 4.398 & .040 & .059 & 3.977 & .050 & .054 & 7.246 & .009 & .094 \\
\hline $\begin{array}{l}\text { Constitución } \\
\text { familiar }\end{array}$ & .864 & .426 & .024 & 1.077 & .346 & .030 & 1.409 & .251 & .039 \\
\hline TIP & 6.061 & .016 & .080 & 1.534 & .220 & .021 & 7.890 & .006 & .101 \\
\hline EVC & 2.938 & .091 & .040 & .025 & .874 & .000 & 2.939 & .091 & .040 \\
\hline Sexo x TIP & .674 & .414 & .010 & .382 & .539 & .005 & 1.215 & .274 & .017 \\
\hline Sexo $x$ EVC & .542 & .464 & .008 & .159 & .691 & .002 & .951 & .333 & .013 \\
\hline $\begin{array}{l}\text { Edad en cate- } \\
\text { gorías x TIP }\end{array}$ & .380 & .540 & .005 & .006 & .940 & .000 & .555 & .459 & .008 \\
\hline $\begin{array}{l}\text { Edad en } \\
\text { categorías x } \\
\text { EVC }\end{array}$ & 1.969 & .165 & .027 & .001 & .980 & .000 & 1.943 & .168 & .027 \\
\hline $\begin{array}{l}\text { Constitución } \\
\text { familiar x TIP }\end{array}$ & .214 & .808 & .006 & .669 & .516 & .019 & .025 & .976 & .001 \\
\hline $\begin{array}{l}\text { Constitución } \\
\text { familiar x } \\
\text { EVC }\end{array}$ & .121 & .886 & .003 & 1.168 & .317 & .032 & .563 & .572 & .016 \\
\hline
\end{tabular}

En cuanto a la escala total del HSCL-A, el modelo explica el $39.7 \%$ de la varianza de la muestra. La edad en categorías $(F(1,85)$ $\left.=7.246 ; p=0.009 ; \eta^{2}=0.094\right)$ y el TIP $\left(F(1,85)=7.89 ; p=0.066 ; \eta^{2}=0.101\right)$ tienen un efecto directo sobre dicha escala. En cuanto a la variable edad, se encontró que los adolescentes en la categoría de 12 a 15 años presentan significativamente menos síntomas psicológicos que los de 16 a 18 años $(B=-2.61 ; S E=0.097 ; t=-2.692$; $p=0.009$ ). Respecto del efecto directo del impacto subjetivo, cuanto más aumenta el impacto, más aumenta la probabilidad de presentar problemas psicológicos $(B=$ $0.178 ; S E=0.088 ; t=2.022 ; p=0.047)$.
Para la escala internalizante, el modelo explica $32.6 \%$ de la varianza en la muestra. Las variables sexo $(F(1,85)=4.082$; $\left.p=0.047 ; \eta^{2}=0.055\right)$, edad en categorías $\left(F(1,85)=4.398 ; p=0.040 ; \eta^{2}=0.059\right)$ $\mathrm{y} \operatorname{TIP}\left(F(1,85)=6.061 ; p=0.016 ; \eta^{2}=\right.$ 0.080 ), tienen un efecto directo -aunque relativamente débil- sobre los puntajes de la subescala internalizante. En concreto, los varones presentan menos problemas internalizantes $(B=-0.339 ; S E=0.168 ; t$ $=-2.02 ; p=0.047)$, y los adolescentes de 12 a 15 años presentan menos problemas internalizantes $(B=-0.265 ; S E=0.126 ; t$ $=-2.097 ; p=0.040)$ que sus pares mayores. En cuanto al TIP, a medida que el mismo 
aumenta, también lo hace la probabilidad de presentar problemas internalizantes $(B=$ $0.179 ; S E=0.115 ; t=1.556 ; p=0.124)$.

Por otro lado, en lo que respecta a la escala externalizante, el modelo explica $37.8 \%$ de la varianza en la muestra. La variable edad resulta la única con un valor predictivo significativo sobre la variable dependiente, a través de un efecto directo $\left(F(1,85)=3.977 ; p=0.050 ; \eta^{2}=0.054\right)$, siendo menores los puntajes obtenidos para problemas externalizantes en el grupo de adolescentes de 12 a 15 años frente a los de 16 a 18 años $(B=-0.247 ; S E=$ $0.124 ; t=-1.994 ; p=0.050)$.

\section{Discusión}

A pesar del vínculo establecido entre la exposición a la violencia en adolescentes y problemas internalizantes, externalizantes y síntomas de estrés postraumático a corto y largo plazo, se han producido pocos datos acerca del tema en Latina América, no obstante las altas tasas de crímenes registradas en los países de este continente. Los únicos datos disponibles para Uruguay sugieren que el $24.6 \%$ de los alumnos de secundaria de entre 14 a 18 años ha sido víctima de robo, agresión o violencia sexual en el último año (Trajtenberg y Eisner, 2015). Estudios internacionales han mostrado que las tasas de exposición suelen ser aún mayores en zonas de bajos recursos (Fowler et al., 2009; Gustafsson et al., 2009).

Por lo tanto, uno de los objetivos de este estudio ha sido la exploración de la exposición a actos de violencia comunitaria en una muestra de adolescentes uruguayos pertenecientes a un contexto socioeconómico desfavorecido en la zona metropolitana de Montevideo. Por otro lado, se pretendía estudiar su relación con la manifestación de problemas internalizantes (síntomas de depresión y ansiedad) y externalizantes (abuso de sustancias, conductas disociales y negativistas desafiantes) en los mismos, considerando el rol mediador de ciertas variables (composición familiar, sexo, edad), y con particular interés en el papel que tiene el impacto percibido de los eventos sobre la prevalencia de dichos problemas psicológicos.

\section{Exposición a la violencia comunitaria e} impacto percibido

Se encontró que el $84.2 \%$ de la muestra había sido expuesto a la violencia comunitaria de forma directa (como víctima) o indirecta (como testigo), $45.6 \%$ de ellos reportando varios eventos a la vez. Aunque no se trata de una muestra representativa para toda la población, los datos nos permiten una primera visión acerca de la amplitud del problema, aún en un país considerado como uno de los menos violentos en la región (UNODC, 2014).

Aunque en la literatura se ha establecido de forma consistente que los adolescentes de mayor edad por lo general perciben tasas más elevadas de exposición a la violencia comunitaria en comparación con sus pares menores (Fowler et al., 2009; Lambert et al., 2005), no se confirmó esta tendencia en la muestra, ni al nivel de su impacto percibido. Aunque se pudo observar niveles de exposición levemente aumentados en los adolescentes de 16 a 18 años en la muestra, no resultaron ser significativamente diferentes de los niveles de exposición del grupo de 12 a 15 años. Es importante mencionar que en este estudio se evaluaron los eventos violentos que cada adolescente ha experimentado en los últimos 12 meses, no a lo largo de toda su vida, lo cual posiblemente explique la ausencia de una relación significativa entre edad y EVC en este estudio (Kennedy y Ceballo, 2014). 
Por otro lado, se confirmó en la muestra la mayor exposición a la violencia comunitaria de varones frente a mujeres, generalmente avalado por la literatura (Javdani et al., 2014); una diferencia que también se reflejó en los niveles del impacto percibido reportado. No obstante, se debe tomar en cuenta la infrarrepresentación de mujeres en la muestra, lo cual exige cautela en la interpretación de los resultados.

En cuanto al papel de la composición familiar en la exposición a la violencia, no se encontraron diferencias significativas entre grupos. Aunque la literatura sugiere un papel protector de mayores niveles de monitoreo, control e involucramiento parental generalmente presente en familias nucleares (Hartinger-Saunders et al., 2011), en la muestra de este estudio no se observaron diferencias significativas en cuanto a las tasas de exposición a la violencia, ni en cuanto a su impacto percibido, en comparación con familias monoparentales o extendidas. Una posible explicación se podría encontrar en las características de la muestra, comprendiendo únicamente jóvenes de nivel socioeconómico bajo. En este contexto desfavorecido, se puede asumir que existen tensiones y estresores que afectan a todas las familias, independientemente de su composición, neutralizando el efecto protector esperado de la familia nuclear (Salzinger et al., 2002).

Respecto de la relación entre la exposición reportada y su impacto percibido, se constata una gran coincidencia entre las dos variables, demostrada a través de una fuerte y significativa correlación ( $r=$ 0.816) entre ellas. Además, el evento más frecuentemente reportado por los adolescentes en la muestra -en este caso el único ítem en la lista de cuatro eventos que indaga acerca de la exposición por testificación de violencia cometida contra otros- resulta ser a la vez el evento con mayor promedio de impacto subjetivo reportado. Este resultado puede considerarse sorprendente, ya que se asume que la violencia atestiguada tiene un impacto psicológico inferior a los eventos experimentados de forma directa y próxima, o sea, como víctima (Fowler et al., 2009).

Dos explicaciones se presentan en este sentido. Por un lado, hay evidencias recientes que cuestionan la utilidad de la categoría violencia atestiguada en su formato único, sin distinguir entre las víctimas de la violencia observada. El estudio de Javdani et al. (2014) muestra que el impacto psicológico de la exposición indirecta como víctima, suele ser mayor cuando se trata de violencia cometida contra familiares, pero solamente en el caso de mujeres adolescentes. Al no haber diferenciado en este estudio el concepto de la exposición a la violencia atestiguada en función de sus víctimas (conocido o desconocido), no es posible saber si su mayor impacto se explica por algún tipo de violencia atestiguada en particular.

Por otro lado, el gran impacto percibido de la violencia atestiguada en esta muestra, puede reflejar el impacto que generan estos eventos a nivel de la comunidad. Se ha establecido que en contextos socioeconómicamente desfavorecidos, la violencia comunitaria suele ser un problema crónico y generalizado, afectando a su población no solamente a través de su efecto potencialmente traumático sobre el funcionamiento psicológico de sus miembros, sino también a través de los sentimientos de inseguridad y desesperanza que genera (Kennedy y Ceballo, 2014; Salzinger et al., 2002). Los elevados puntajes de impacto percibido para los eventos atestiguados pueden estar reflejando justamente estas inquietudes, fortalecidas por los medios, la opinión pública y política, etc. (Guerra et al., 2003). 
Predictores de problemas psicológicos en situación de violencia comunitaria

Explorando la relación bivariada entre la exposición a la violencia comunitaria y su impacto percibido por un lado, y problemas psicológicos por otro lado, se mostró una considerable correlación de los primeros con problemas externalizantes. Los problemas internalizantes reportados parecían correlacionarse solamente con el impacto percibido de la violencia, y no con la exposición real. Estos datos parecen coincidir con los de Hill y Madhere (1996).

No obstante, a pesar de las tendencias que sugieren estos datos, para poder considerar adecuadamente la contribución de cada una de las variables de interés, se llevó a cabo una ANCOVA, evaluando el valor predictor de ellas sobre los problemas psicológicos reportados de forma integral. Las variables edad e impacto percibido de la violencia comunitaria aparecen como principales predictores para los puntajes totales del HSCL-A y su subescala de problemas internalizantes. La mayor edad se asocia con mayor cantidad de problemas psicológicos de ambos tipos, tendencia que se ha explicado por la mayor capacidad cognitiva de los adolescentes mayores para darse cuenta de las implicancias perturbadores de la violencia para su propia vida, las de sus seres queridos y para la sociedad en general (Grant et al., 2005). Consistentemente con la literatura, se constata un efecto directo de género sobre la sintomatología internalizante, siendo mayor para mujeres (Javdani et al., 2014), aunque en este estudio en particular no se le da mucho protagonismo a la variable, por temas de sesgo en la muestra.

En cuanto a la discusión acerca del efecto diferencial de la exposición a la violencia y su impacto percibido en la sintomatolo- gía producida, los datos del estudio apoyan la teoría de evaluación cognitiva (Lazarus y Folkman, 1984), demostrando la contribución significativa del impacto percibido sobre todo en cuanto a síntomas de depresión y ansiedad. Se considera que estos síntomas pueden ser otra manifestación más de la perturbación psicológica o el trauma generado por la exposición a eventos de violencia, considerados como impactantes por los sujetos (Mels et al., 2010b; Miller y Rasmussen, 2010). No obstante, la ausencia de un efecto de la exposición real sobre los síntomas evaluados no implica que sea una variable irrelevante, sobre todo considerando la importante correlación encontrada entre la exposición acumulada y el impacto total percibido.

Sorprendentemente, investigando los predictores de la sintomatología externalizante en esta muestra, solamente se evidenció un efecto directo significativo de la variable edad, como si operara de forma independiente de la violencia vivida. Como ya se mencionó anteriormente, el mayor grado de problemas externalizantes en adolescentes mayores está establecido y hasta considerado como normativo en esta etapa del desarrollo humano (Achenbach y Edelbrock, 1978). La cultura adolescente, las conductas de riesgo vinculadas a la adolescencia y las tensiones que genera la búsqueda de identidad e independencia en esta etapa, llevan a una mayor manifestación de conductas oposicionistas y transgresiones de normas (Aisenberg y Herrenkohl, 2008). No obstante, en la literatura especializada, la sintomatología externalizante presente en adolescentes en situación de violencia comunitaria suele ser atribuida en gran medida a sus niveles de exposición (Fowler et al., 2009). ¿Por qué entonces no es así en la muestra de este estudio? 
Una posible explicación se encuentra en la teoría del aprendizaje social, frecuentemente utilizada para explicar los efectos de la violencia comunitaria sobre conductas externalizantes y delincuencia en adolescentes (Guerra et al., 2003). Dicha teoría estipula que estas conductas se reproducen a través de efectos de modelaje y aprendizaje vicario en la comunidad, los cuales según la teoría socioecológica (Bronfenbrenner, 1979) se transponen a nivel de las interacciones en la familia y entre pares (Salzinger et al., 2002). Se diferencia entonces de los modelos de trauma, implicando la exposición directa o indirecta a eventos violentos particulares como el principal motor para el desarrollo de problemas psicológicos. Aunque muchos estudios han demostrado un vínculo entre la EVC y problemas de conducta, el aprendizaje social en sociedades con altas tasas de violencia supuestamente se da a través de una influencia casi constante de modelos de interacción y de conducta presentes y reproducidos en la vida diaria (Javdani et al., 2014), y no necesariamente a través de la vivencia de "eventos impactantes".

Por último, la ausencia de un efecto de la constitución familiar sobre los problemas psicológicos reportados por los adolescentes (controlados por otras variables relevantes en este estudio), se deja explicar por la composición de la muestra. Por la exclusiva inclusión de adolescentes pertenecientes a contextos socioeconómicamente desfavorecidos, se puede asumir que los factores protectores por lo general asociados con la familia nuclear (grado de control, estabilidad, la presencia de modelos positivos), se ven negativamente afectados por las condiciones de vida en esta población (HartingerSaunders et al., 2011).

\section{Limitaciones del estudio e implicacio-} nes para el futuro

Varias limitaciones del presente estudio deben ser tomadas en consideración a la hora de interpretar sus resultados. Aunque ciertos autores han demostrado la buena fiabilidad de los cuestionarios de autorreporte en población adolescente (Bean et al., 2007), se considera como una limitación de esta investigación el no haber incluido varios informantes o técnicas de estudio para corroborar el autoinforme de síntomas en los adolescentes en la muestra. Se reitera que la poca cantidad de mujeres en el estudio obstaculiza la generalización de los resultados encontrados en base a género. Asimismo, la muestra fue seleccionada por conveniencia por conglomerado, no al azar, lo cual entorpece la generalización de los datos a la población general.

Se considera que el estudio cuantitativo de la realidad siempre constituye una reducción de la misma, dejando de lado la implicación de diversos factores importantes en el estudio. El valor de las varianzas explicadas por los modelos ANCOVA investigados en este estudio (variando entre el $32.6 \%$ y $39.7 \%$ ) confirman esta aseveración. Por otro lado, de acuerdo con Kennedy y Ceballo (2014) se reconocen las limitaciones de los instrumentos usados para la evaluación de la exposición a la violencia en esta muestra, su impacto percibido y su incapacidad de investigar el impacto diferencial de varias dimensiones relevantes de la exposición a la violencia comunitaria. Es necesario a futuro que se sigan investigando las características psicométricas de los instrumentos usados en este trabajo, ampliando los conocimientos respecto de su fiabilidad y validez. 
No obstante, al tratarse de uno de los primeros estudios en el país y la región en cuanto a los efectos psicológicos de la violencia comunitaria en adolescentes, un fenómeno de creciente interés para la salud pública, sobre todo en áreas urbanas, este trabajo - a pesar de sus limitaciones- genera datos relevantes que permiten avanzar en propuestas preventivas.

El papel destacado del impacto subjetivo de la violencia comunitaria sobre la manifestación de problemas psicológicos, brinda una herramienta de prevención importante, aún tras la exposición sufrida. Abre el camino para el uso de psicoeducación en esta población, para generar una mayor conciencia acerca de la propia influencia del sujeto en su bienestar psicológico y su poder de cambio. En la misma línea, las intervenciones grupales que generan un espacio de discusión en torno a la violencia comunitaria sufrida por los integrantes tienen el potencial de cambiar la perspectiva de la víctima, a la hora de contrastar sus experiencias con las de otros (Thomas et al., 2012). Cada intervención que logra cambiar la percepción del impacto subjetivo de los eventos sufridos puede, eventualmente, reducir sus efectos negativos sobre la salud mental (Hill y Madhere, 1996).

La evidencia que genera el estudio en cuanto a la importancia de los factores de riesgo a nivel personal permite plantear sugerencias respecto de hacia dónde dirigir los recursos preventivos disponibles. El grupo de adolescentes de 16 a 18 años en particular podría beneficiarse de actividades de canalización -deporte, música y teatro, entre otros- en un intento de reducir conductas externalizantes. No obstante, desde el enfoque de la teoría del aprendizaje social, dichas actividades tendrían que estar acompañadas por otras que trabajen la identificación con modelos y patrones de interacción agresivos en la sociedad, y su competencia para generar alternativas a través de sus propias conductas.

Salzinger et al. (2002) plantean que, en cuanto a las consecuencias psicológicas y académicas de la exposición a la violencia comunitaria en niños y adolescentes, la escuela/ liceo "es un escenario heurístico en el cual la intervención o programas preventivos tendrían que estar situados para aumentar su probabilidad para ser exitoso" (p. 445). Siguiendo a las ideas de Bronfenbrenner (1979) acerca de la interconectividad de los contextos de desarrollo humano, surge la necesidad de hacer participar no solamente a los maestros y otro tipo de personal educativo en su abordaje, sino también a los familiares de los adolescentes, para que todos conozcan los efectos nocivos de la violencia, los mecanismos de su reproducción y su propio papel en ellos. No obstante, los centros educativos pueden ser un buen lugar para empezar el abordaje de esta problemática que concierne a toda la sociedad.

\section{Referencias}

Achenbach, T. M. \& Edelbrock, C. S. (1978). The classification of child psychopathology: A review and analysis of empirical efforts. Psychological Bulletin, 85(6), 1275-1301. Retrieved from http://psycnet.apa.org/index.cfm?fa=buy.optionToBuy\&id=1979-26083-001 
Aisenberg, E. \& Herrenkohl, T. (2008). Community violence in context: Risk and resilience in children and families. Journal of Interpersonal Violence, 23, 296-315. http://dx.doi.org/10.1177/0886260507312287

Bean, T., Derluyn, I., Eurelings-Bontekoe, E., Broekaert, E., \& Spinhoven, P. (2007). Validation of the multiple language versions of the Hopkins Symptom Checklist-37 for Refugee Adolescents. Adolescence, 42, 51-71.

Borofsky, L., Kellerman, I., Baucom, B., Oliver, P., \& Margolin, G. (2013). Community violence exposure and adolescents' school engagement and academic achievement over time. Psychology of Violence, 3(4), 381-395.

http://dx.doi.org/10.1037/a0034121

Bowen, N. \& Bowen, G. (1999). Effects of crime and violence in neighborhoods and schools on the school behavior and performance of adolescents. Journal of Adolescent Research, 14(3), 319-342.

http://dx.doi.org/10.1177/0743558499143003

Bronfenbrennner, U. (1979). La ecología del desarrollo humano. Experimentos en entornos naturales y diseñados. Barcelona: Paidós.

Cooley-Strickland, M., Quille, T., Griffin, R., Stuart, E., Bradshaw, C., \& Furr-Holden, D. (2009). Community violence and youth: Affect, behavior, substance use, and academics. Clinical Child and Family Psychological Review, 12, 127-156.

http://dx.doi.org/10.1007/s10567-009-0051-6

Copeland-Linder, N., Lambert, S., \& Ialongo, N. (2010). Community violence, protective factors and adolescent mental health: A profile analysis. Journal of Clinical Child \& Adolescent Psychology, 39(2), 176-186.

Fowler, P., Tompsett, C., Braciszewski, J., Jacques-Tiura, A. J., \& Baltes, B. B. (2009). Community violence: A meta-analysis on the effect of exposure and mental health outcomes of children and adolescents. Development \& Psychopathology, 21, 227-259.

Gorman-Smith, D. \& Tolan, P. (1998). The role of exposure to community violence and developmental problems among innercity youth. Development and Psychopathology, 10, 101-116.

Grant, K. E., McCormick, A., Poindexter, L., Simpkins, T., Janda, C. M., Thomas, K. J., ... Taylor, J. (2005). Exposure to violence and parenting as mediators between poverty and psychological symptoms in urban African American adolescents. Journal of Adolescence, $28,507-521$.

http://dx.doi.org/10.1016/j.adolescence.2004.12.001

Guerra, N. G., Huesmann, L. R., \& Spindler, A. J. (2003). Community violence exposure, social cognition, and aggression among urban elementary-school children. Child Development, 74(5), 1561-1576.

http://dx.doi.org/10.1111/1467-8624.00623

Gustafsson, P., Larsson, I., Nelson, N., \& Gustafsson, P. A. (2009). Sociocultural disadvantage, traumatic life events, and psychiatric symptoms in preadolescent children. American Journal of Orthopsychiatry, 79, 387-397.

http://dx.doi.org/10.1037/a0016559 
Hartinger-Saunders, R. M., Rittner, B., Wieczorek, W., Nochajski, T., Rine, C. M., \& Welte, J. (2011). Victimization, psychological distress, and subsequent offending among youth. Children and Youth Services Review, 33, 2375-2385.

http://dx.doi.org/10.1016/j.childyouth.2011.08.009

Hill, H. \& Madhere, S. (1996). Exposure to community violence and African American children: A multidimensional model of risks and resources. Journal of Community Psychology, 24, 26-43.

Horowitz, K., McKay, M., \& Marshall, R. (2005). Community violence and urban families: Experiences, effects, and directions for intervention. American Journal of Orthopsychiatry, 75, 356-368.

http://dx.doi.org/10.1037/0002-9432.75.3.356

Instituto Nacional de Estadística, INE. (2013). Estimación de la pobreza por el método del Ingreso - Año 2012. Montevideo: Autor.

Javdani, S., Abdul-Adil, J., Suárez, L., Nichols, S. R., \& Farmer, A. D. (2014). Gender differences in the effects of community violence on mental health outcomes in a sample of low-income youth receiving psychiatric care. American Journal of Community Psychology, 53(3-4), 235-248.

http://dx.doi.org/10.1007/s10464-014-9638-2

Kennedy, T. M. \& Ceballo, R. (2014). Who, what, when, and where? Toward a dimensional conceptualization of community violence exposure. Review of General Psychology, 18(2), 69-81.

http://dx.doi.org/10.1037/gpr0000005

Lambert, S. F., Ialongo, N. S., Boyd, R. C., \& Cooley, M. R. (2005). Risk factors for community violence exposure in adolescence. American Journal of Community Psychology, $36,29-48$.

http://dx.doi.org/10.1007/s10464-005-6231-8

Lazarus, R. S. \& Folkman, S. (1984). Stress, appraisal, and coping. New York: Springer Publishing.

Leiner, M., Puertas, H., Caratachea, R., Ávila, C., Atluru, A., Briones, D., \& de Vargas, C. (2012). Children's mental health and collective violence: A binational study on the United States-Mexico border. Revista Panamericana de Salud Pública, 31(5), 411-416.

http://dx.doi.org/10.1590/S1020-49892012000500009

Ludwig, K. \& Warren, J. (2009). Community violence, school-related protective factors, and psychosocial outcomes in urban youth. Psychology in the Schools, 46(10), 1061-1073. http://dx.doi.org/10.1002/pits.20444

Luthar, S. S. \& Cicchetti, D. (2000). The construct of resilience: Implications for interventions and social policies. Developmental Psychopathology, 12(4), 857-885.

http://dx.doi.org/10.1017/S0954579400004156

Lynch, M. (2003). Consequences of children's exposure to community violence. Clinical Child and Family Psychology Review, 6(4), 265-274.

http://dx.doi.org/10.1023/B:CCFP.0000006293.77143.e1 
Mels, C. (2012). Entre pobreza y violencia: ¿cómo afectan los estresores diarios y la violencia a adolescentes en zonas de guerra? Ciencias Psicológicas, 6(2), 111-122. Recuperado de http://www.scielo.edu.uy/scielo.php?pid=S1688-42212012000200002\&script=sci_arttext

Mels, C., Derluyn, I., Broekaert, E., \& Rosseel, Y. (2009). Traumatic exposure and posttraumatic stress symptoms in adolescents in war-affected Eastern Democratic Republic of Congo. Archives of Pediatrics \& Adolescent Medicine, 163(6), 525-530.

Mels, C., Derluyn, I., Broekaert, E., \& Rosseel, Y. (2010a). The psychological impact of forced displacement and related risk factors on Eastern Congolese adolescents affected by war. Child Psychology \& Psychiatry, 51(10), 1096-1104.

http://dx.doi.org/10.1111/j.1469-7610.2010.02241.x

Mels, C., Derluyn, I., Broekaert, E., \& Rosseel, Y. (2010b). Community-based crosscultural adaptation of mental health measures in emergency settings: Validating the IES$\mathrm{R}$ and HSCL-37A in Eastern Democratic Republic of Congo. Social Psychiatry \& Psychiatric Epidemiology, 45, 899-910.

http://dx.doi.org/10.1007/s00127-009-0128-z

Mels, C. y Trías, D. (2014). Características psicométricas preliminares del HSCL-A adaptado para adolescentes uruguayos en contexto de violencia. Ciencias Psicológicas, $7(2), 139-149$.

Miller, K. E. \& Rasmussen, A. (2010). War exposure, daily stressors, and mental health in conflict and post-conflict settings: Bridging the divide between trauma-focused and psychosocial frameworks. Social Science \& Medicine, 70(1), 7-16.

http://dx.doi.org/10.1016/j.socscimed.2009.09.029

Munyo, I. (2012). Los dilemas de la delincuencia juvenil en Uruguay. Montevideo: Centro de Estudios de la Realidad Económica y Social.

Organización Mundial de la Salud, OMS. (2003). Informe sobre la salud en el mundo: forjemos el futuro. Ginebra: Autor.

Osypuk, T., Schmidt, N., Bates, L., Tchetgen-Tchetgen, E., Earls, F., \& Glymour, M. (2012). Gender and crime victimization modify neighborhood effects on adolescent mental health. Pediatrics, 130(3), 472-481.

http://dx.doi.org/10.1542/peds.2011-2535

Paternain, R. y Sanseviero, R. (2008). Violencia, inseguridad y miedos en Uruguay. ¿Qué tienen para decir las ciencias sociales? Montevideo: Friedrich Ebert Stiftung.

Rosenfeld, L., Richman, J., Bowen, G., \& Wynns, S. (2006). In the face of a dangerous community: The effects of social support and neighborhood danger on high school student's school outcomes. Southern Communication Journal, 71(3), 273-289.

http://dx.doi.org/10.1080/10417940600846045

Saltzman, H., Compas, B. E., Connor-Smith, J. K., Harding Thomsen, A., \& Wadsworth, M. E. (2001). Coping with stress during childhood and adolescence: Problems, progress, and potential in theory and research. Psychological Bulletin, 17, 87-127.

http://dx.doi.org/10.1037//0033-2909.127.1.87 
Salzinger, S., Feldman, R. S., Stockhammer, T., \& Hood, J. (2002). An ecological framework for understanding risk for exposure to community violence and the effects of exposure on children and adolescents. Aggression and Violent Behavior, 7, 423-451. http://dx.doi.org/10.1016/S1359-1789(01)00078-7

Seiffge-Krenke, I. (2000). Causal links between stressful events, coping style and adolescent symptomatology. Journal of Adolescence, 23, 675-691.

http://dx.doi.org/10.1006/jado.2000.0352

SPSS Inc. (2009). PASW Statistics for Windows, Version 18.0. Chicago: SPSS Inc.

Steinbrenner, S. (2010). Concept analysis of community violence: Using adolescent exposure to community violence as an exemplar. Issues in Mental Health Nursing, 31, 4-7. http://dx.doi.org/10.3109/01612840903200050

Trajtenberg, N. y Eisner, M. (2015). Hacia una política de prevención de la violencia en Uruguay. Montevideo/Cambridge: University of Cambridge, UDELAR, ANEP.

Thomas, A., Carey, D., Prewitt, K. R., Romero, E., Richards, M., \& Velsor-Friedrich, B. (2012). African-American youth and exposure to community violence: Supporting change from the inside. Journal for Social Action in Counseling and Psychology, 4(1), 54-68. Retrieved from http://www.psysr.org/jsacp/Thomas-v4n1-12_54-68.pdf

United Nations Office on Drugs and Crime, UNODC (2014). Global study on homicide 2013. Trends, contexts, data. Vienna: United Nations Office on Drugs and Crime. 